\title{
MINING OF IMAGES USING RETRIEVAL TECHNIQUES
}

\author{
Pooja B. Shaha ${ }^{1}$, Malvika U. Saraf ${ }^{2}$ \\ ${ }^{1}$ M.Tech. Scholar, Department of Electronics and Telecommunication, Wainganga College of Engineering and \\ Management, Nagpur, Maharashtra, India \\ ${ }^{2}$ Head of the Department, Department of Electronics and Telecommunication, Wainganga College of Engineering and \\ Management, Nagpur, Maharashtra, India
}

\begin{abstract}
Today's world is digital. Use of different social websites is become a part of our day to day life. These social websites have acquired great popularity because of their user-friendly features and their content. Nowadays internet and mobile networks are widely used everywhere and so use of images. Because of evolution in hardware as well as software it is possible to store large amount of multimedia data. In short, now a day it becomes easy to store huge amount of images by using image processing techniques. As number of images and databases are increasing day by day, there is a need for new image retrieval techniques that should be fulfilled. Image mining is derived from data mining which is a method to extract information from digital image. The purpose of this paper is to present a review of the various image mining techniques used in different applications as image retrieval, Matching, Pattern recognition etc.given by different researchers. An information or knowledge can be extracted from the image by using image mining technique. This paper proposes the survey in image retrieval techniques. Image retrieval and data mining have huge applications in the sector of image processing, pattern recognition, image matching, image mining, feature extraction, computer vision, etc.
\end{abstract}

Keywords : Image, Image Mining, Feature Extraction, Image Retrieval CBIR.

\section{INTRODUCTION}

Before 90's images can be searched over the internet using textual information only. No doubt, today also we are using the same for finding images on web. But nowadays contentbased information can also used to retrieve image from a data basealong with textual information. An image retrieval system is nothing but a computer systembuild for finding and mining images from a hugedatabase of digital images.Image mining is nothing but extracting knowledge from an image by extracting its features.

\subsection{Difference between Image Mining and Data}

\section{Mining:}

Mining of an image does not mean to apply respective steps or algorithm to get specific image. It differsfrom mining of data by some features. Few facts regarding image and data mining can be discussed here:

(1) An image can be represented in terms of some values (pixel values) which are irrelevant to the content (subject) of an image. But for a text or data mining, the numbers or values are relevant to actual content of data.

(2) Another important difference regarding image and data mining is there may be misinterpretation of an image by image retrieval system. But data mining system may give closely matching result for mining. For e.g., if an image of an apple is given in input for mining, the result may obtained as red apples as well as green apples. So, there is a need to build a system which can minimize this problem [2].
A well known knowledge claim is that asingle image can replace thousands of words. Unluckily, these thousands ofwords may differ from one individual to another depending on their perspective and/or knowledge of the image context.[11] For example,

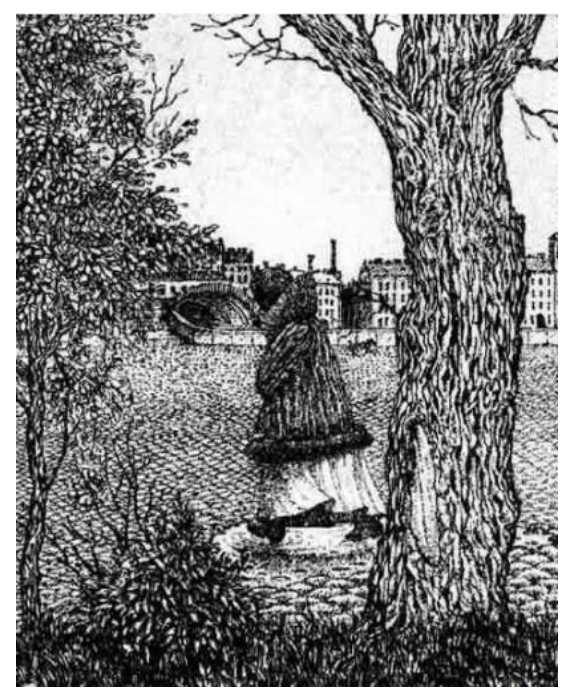

Fig.1 Illusion

Above fig. may come under the category of tree or city or human face or even a statue. It gives a familiar demonstration that an image can have multiple, quite different interpretations. [10] Thus, even if a 1000-word image description were available, it is not sure that the image could be retrieved by a user with some other explanations. 
The basic difficulty found in conveying an information or image to a user and the image retrieval system. It may possible that the user may be in need of different knowledge about image database. So the retrieval system should be build in a way such that it can be answerable to various forms of query. Generally image retrieval queries can come under following categories:

1. Attribute-based - Uses context, structural metadata.

2. Textual- Uses a term-based specification of input images that can be matched to textual image descriptors.

3. Visual-Give visual characteristics (color, texture) or an image which can be compared to visual descriptors.[12]

The sensory gap is the difference between the real worldobject and the informationin a (computational) description taken from a database of that object.

The semantic gap is the difference between the information thatone can extract from the visual data and the explanation that the same data has for a user in a given situation. [11]

The biggest task in CBIR is to reduce these gaps to improve performance of image retrieval system. The further part of this paper explains the different techniques used for image mining in section 2 and work done previously related to this topic in section 3 .

\section{IMAGE MINING TECHNIQUES}

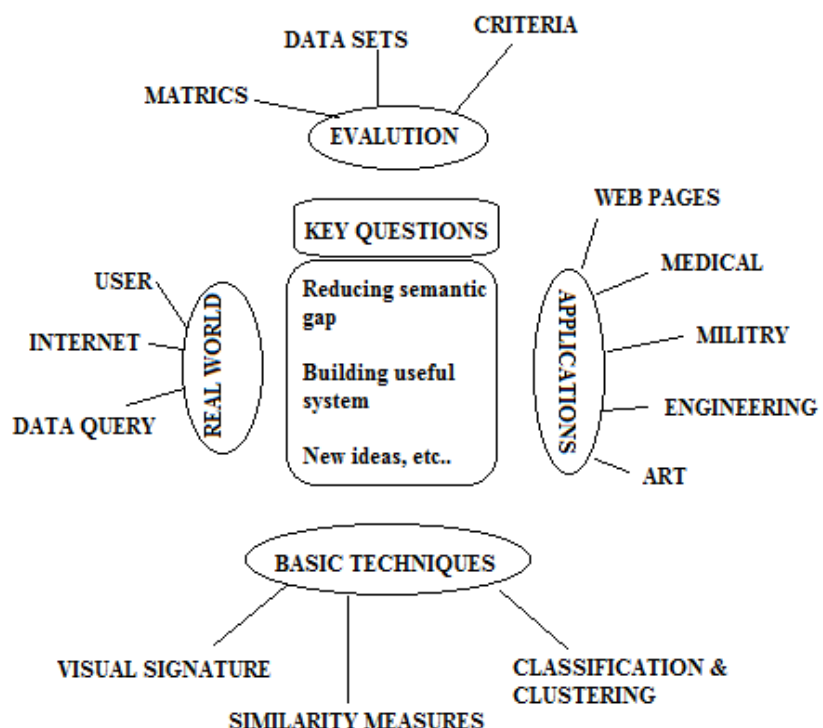

Fig.2 Factors related to image mining. [11]

\subsection{Object Recognition}

Object recognition is the way to tell an object's physical properties (viz. shape, color and texture) and tell meaningful attributes to the object, which involves the perception of its use, earlier experience with the object and its relation with others. The information can be extracted if and only if the object is recognized correctly. In short, object recognition is assigning proper tag to correct parts of an image. [2]

\section{Object Recognition}
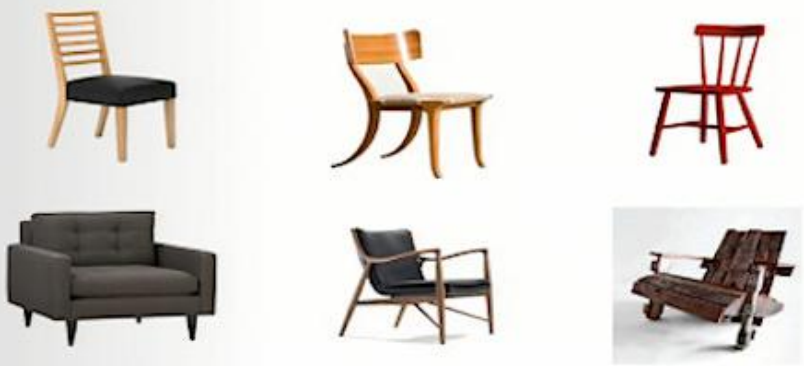

Recognize that these objects are chairs..

Fig.3 Object Chair is recognized.

\subsection{Image Retrieval}

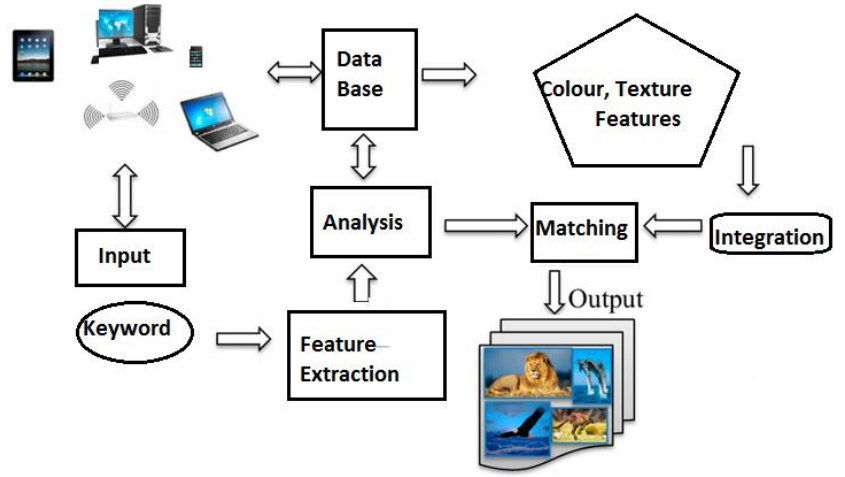

Fig.4 Basic stages involved in image retrieval

\section{Text Based Image Retrieval}

Text-based image retrieval based on traditional database techniques for organizing images. An image can be described in text by categorizing under different topics or in hierarchical format so that finding becomes easy. But every time this will not be done automatically. Sometimes it is required to comment manually for images. [3]

\section{Query Based Image Retrieval}

Giving image as an input query- query based image retrieval system searches for the best matching image for query image from its database and compares the obtained result with query image. But this process is useful only when both, query image and obtained image are of same type of database like painting-painting image database [9].

\subsection{Image Indexing}

Image indexing is the technique to find similar images of the requested input image from its database. Image indexing can be done by comparing features viz. color, texture, structure, etc. of input image with images from database. Image indexing is of following two types-

1. Textual: It is very simple technique. The keyword includes are heading indexing, typical content or grouping etc. 
2. Content Based: Content-based image retrieval uses visual description for finding image from a database as per the request of user. [3]

In CBIR, visual description from the database is explained in multi-dimensional feature vectors after extraction. The feature database can be formed by feature vectors of images in database. User will provide an image to the retrieval system for extracting the matching image. The system will extract the features of input image by the user and will arrange them in the form of vectors. The query image feature vectors will be compared with the currently present feature vectors in database for retrieving purpose by using indexing technique. An Indexing technique is an efficient method for finding an image database. As per the new developments in retrieval techniques, more correct results can be found based on user acknowledgement. [4]

\subsection{Image Classification and Image Clustering}

Images can be classified under two groups as supervised and unsupervised classes. Supervised group is a pre labelled group. So it becomes very tough to add any new image which is not labelled previously. But in unsupervised, adding an unlabelled image to any cluster without any background of that image is a big task. [2]

1. Image Texture Classification- Energy of an image is represented by texture. The energy will be high if image contains high texture compared to mean and low texture images. In short, energy values are high for high texture images and low for low texture images. This is called as "Texture Activity Index". [2] Because of this indexing, it become very easy and effective to classify images under high, low, average description because of statistical parameters.

2. Image Clustering- One of the benefits of clustering is that it reduces the searching time for an image from database. There are several clustering techniques available as: hierarchal, partitioning, density-based, grid based and fuzzy clustering methods. Fuzzy C-means (FCM) is one of the clustering methods used frequently in mining of an image. This technique allows one piece of data to connect to 2 or more than 2 clusters. Instead of connection to single cluster in this clustering, there will be a degree of connecting to other clusters. Hence, the points in centre of cluster have more degree compared to points on edges. FCM classifies data in specific number of clusters. [2]

\subsection{Association Rule Mining}

Association rule mining is frequently used in data mining for different patterns, shapes. They can be used for mining an image from large database. There are 2 ways for mining: in first method, data can be mined from single large database only and in second method, there will combine database of alphanumeric data by using association rule mining, patterns can be detect to extract information. This technique is useful to extract information from very large database.

\subsection{Neural Networks}

Neural Networks is again one more method which can be used for mining and processing an image Neural Networks computational systems work on the principal of nervous system in human beings. The basic processing element is known as neuron. These computational blocks work similar to neurons present in our nervous system. They are also having multiple connections to the network. The role of neuron is to receive activation values from other neurons then compute on the obtained result as per their input parameters and give it to next neuron. This artificial neural network (ANN) is very important to study as well as a great field of research as this network system based on human behaviour like structure. Then neural networks are trained by using different training algorithm to achieve desired result. Neural networks differ from conventional networks as conventional networks work on programming but neural networks have training algorithms used with training data. Possibility of faults is less comparatively in case of neural networks.

\section{RELATED WORK}

R. Datta et al. (2002) says, Content-based image retrieval has studied in many fields in recent years. CBIR is advantageous over keyword based retrieval because of its effective management as well as retrieval. The previously used methods were not lagging behind in describing human behavior also expressions. Because of this, getting satisfactory result becomes quite difficult for the image asked by the user. As a solution for the above problem the above authors have designed a system which has a base of human preference and emotion by using an interactive genetic algorithm (IGA). The system developed by above authors is using wavelet transform for extracting image features. The system also gives a user-friendly way for retrieving an image when user is not clear about the idea of image which he wants from a huge database. Thus, this type of searching serves finding for any image with implicit as well as explicit queries. A database with two thousand images is used for the proposed system development. [13]

Nishchol Mishra et al.(2012) says, spread of internet and network sites including social networking sites has been the new scenario of this century. These networking cites are with full of multimedia data that can be mined and analyzed. Extracting knowledge from this huge multimedia data is a very challenging task.

So the new field of research has been opened as Multimedia Data mining (MDM). Multimedia Data Mining is a method of searching interesting frameworks from multimedia data which are generally inaccessible by using simple queries and allies output. This paper has highlighted Image Mining techniques and also tells the importance of CBIR in image mining. [5]

Shaikh Nikhat Fatima (2012) says, the aim of their paper is exploring image patterns which are important from a given set or of images. The main objective is of searching 
low level pixel representation contained in a unprocessed image can be efficiently and effectively processed to recognize high level features. Review on image mining is also Overview of image mining frameworks is also discussed in this paper. The system extracts knowledge from images also considers challenges of getting desired outputs relevant to query images. [6]

M. V. Das et al. (2014) says, use of image mining or image retrieving methods has been increased from last few decades as the use of multi-media data viz. audio, video, images etc. has been increased because of different networking sites. A correctness of image retrieval can be increase by using $\mathrm{A}$ content based image retrieval system (CBIR) is suggested to increase accuracy in image mining by using interactive genetic algorithm (IGA). Low level image descriptors in CBIR are color, texture, edge. In this paper authors have suggested a system that separates the retrieving process in 2 stages. Query stage and evaluation stage. Feature descriptors were extracted of input image and then similarity between input image and images from database are calculated. This is the first stage, query stage. Evolution stage retrieves most similar image using IGA.Use of IGA to help user to know the most satisfied result to user's need. The color image database used for experimental evaluation is 10000WANG. It can be seen that their proposed work is accomplished with the experimental results. [1]

\section{CONCLUSIONS}

There is a huge scope for research in a field of image processing and especially in image mining. In this paper we have discussed the basics of image mining and different techniques used for mining an image from image data base. As per the work done in previous research till date, still the presence of semantic gap can be found. This gap should be minimized. There is a need of designing a strong language for image database to get the optimized and focused result. Our proposed work will be to build a CVS (Computer visual system) system which will be closed to HVS (Human Visual System) by considering their visual complexity and to obtain a focused result for image retrieving system.

\section{REFERENCES}

[1] M. V. Dass et al., "Image Retrieval Using Interactive Genetic Algorithm", Computational Science and Computational Intelligence (CSCI), 2014 International Conference on (Volume:1 ), 10-13 March 2014, 215-220, DOI:10.1109/CSCI.2014.44, IEEE.

[2] PrabhjeetKaur and KamaljitKaur, "Review of Different Existing Image Mining Techniques", International Journalof Advanced Research in Computer Science and Software Engineering 4(6),June -2014, pp.518-524.

[3] Abdullah M. Iliyasu et al., "Mining visual complexity of images based on an enhanced feature spaceRepresentation",978-1-4673-4544-6/13/2013 IEEE.

[4] D. D. Feng et al., "Multimedia information retrieval and management: Technological fundamentals and applications”, Springer Science \& Business Media, 2013, ch. 1.

[5] Nishchol Mishra and Dr. Sanjay Silakari "A Perspective Image Mining in the Context of Content Based Image Retrieval", IJCSI InternationalJournal of Computer Science Issues, Vol. 9, Issue 4, No 3, July 2012.

[6] ShaikhNikhatFatma ," Image Mining Method and Frameworks",International Journal of Computational Engineering Research (ijceronline.com)Vol. Issue. 8, 2012.

[7] G. Duan, J. Yang and Y. Yang, "Content-based image retrieval research", Int. Conf. on Phy Sci. \& Tech. (ICPS, 2011), Physics procedia Vol. 22 (2011), pp. 471-477.

[8] RamadassSudhir, "A Survey on Image Mining Techniques: Theory andApplications", Computer Engineering and Intelligent Systems, ISSN 2222-1719 (Paper) ISSN 2222-2863 (Online) Vol 2, No.6, 2011.

[9] A.kannan, Dr.v.mohan and Dr.N.anbazhagan, "An effective method of image retrieval usingImage mining techniques", the international journal of multimedia \& its applications (ijma) vol.2, no.4, November 2010.

[10] A.Kannan, Dr.V.Mohan and Dr.N.Anbazhagan, "Image Clustering and Retrieval using Image Mining Techniques", 2010 IEEE International Conference on Computational Intelligence and Computing Research.

[11] M. Cardici, V. D. Gesu, M. Petrou, and M. E. Tabacchi,"A Fuzzy Approach to the Evaluation of ImageComplexity", Fuzzy Sets and Systems, pp. 14741484,Vol.160, 2009.

[12] Joan C. Nordbotten, "Multimedia Information Retrieval Systems ”, DRAFT v2.0 Dec.'08, Ch9.

[13] R. Datta et al.," Image Retrieval: Ideas, Influences, and Trends of the New Age", ACM Comput. Surv. 40, 2, Article 5 (April 2008), 60 pages DOI $=10.1145 / 1348246.1348248$ http://doi.acm.org/10.1145/1348246.1348248.

[14] Peter stanchev, "using image mining for image retrieval", iasted conf. Computer science and technology, may 19-20, 2003, cancum, mexico, 214-218.

[15] Sung-Bae-Cho , Joo-Young Lee, "A human-oriented image retrieval system using interactive genetic algorithm", IEEE Transactions on Systems, Man, and Cybernetics - Part A: Systems and Humans (Volume:32, Issue: 3 ), ISSN :1083-4427, DOI:10.1109/TSMCA.2002.802812, May 2002.

[16] Carlos Ordonez and Edward Omiecinski, "Image Mining: A New Approach for Data Mining", College of Computing, Georgia Institute of TechnologyAtlanta, Georgia 30332-0280, February 20, 1998.

[17] G. Pass and R. Zabih, "Histogram refinement forcontent-based image retrieval", Proc. of 3rd IEEE.Workshop on Applications of Computer Vision, WACV, pp.96 - 102, 1996.

[18]J. Dowe, "Content-based retrieval in multimedia imaging," In Proc. SPIE Storage and Retrieval for Image and Video Database, 1993.

[19] MahipM.Bartere and Dr.Prashant,R.Deshmukh," An efficient Technique using Text \& Content Base Image Mining Technique for Image Retrieval “. 


\section{BIOGRAPHIES}

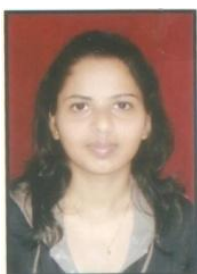

Pooja B. Shaha, M.Tech. Scholar, Department of Electronics and Electronics and Telecommunication, Wainganga College of Engineering and Management, Nagpur, Maharashtra, India.

E-mail id: netrajain1108@gmail.com

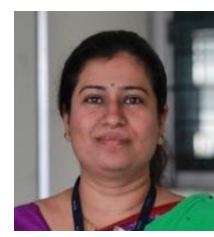

Prof. Malvika U. Saraf, Head of the Department, Department of Electronics and Electronics and Telecommunication, Wainganga College of Engineering And Management, Nagpur, Maharashtra, India. E-mail id: malvika_deole@rediffmail.com 